Journal of Case Reports 2020;10(4):250-253

\title{
Post Snake Bite Compartment Syndrome of Lower Limb in a Child
}

\author{
Deepak Jain, Parimal Malviya, Tushar Agrawal, Alfven Vieira, Ashish Naik, Ananya Sharma, Sharvari \\ Gunjotikar, Shreenath Vishwanath
}

Department of Orthopaedics, MGM Institute of Health Sciences, Navi Mumbai, Maharashtra, India.

\section{Corresponding Author: \\ Dr. Parimal Malviya \\ Email: dr.parima190@gmail.com}

This is an Open Access article distributed under the terms of the Creative Commons Attribution License (creativecommons.org/ licenses/by/3.0).

\begin{abstract}
Background: Compartment syndrome after a snake bite is an uncommon occurrence. The effects are more pronounced in children possibly due to the reduced total dilution volume in children. Case Report: A 10-year-old boy presented with an alleged history of snakebite by a banded krait just above the lateral malleolus. The patient eventually developed compartment syndrome of the right lower limb involving leg and thigh which was diagnosed based on clinical evaluation \& laboratory findings and was managed by emergency fasciotomy. Conclusion: Snake bitten limbs need constant monitoring in a hospital setting for any evolving systemic and local complications. Early surgical intervention may prove to be limb saving.
\end{abstract}

Keywords: Acidosis, Ankle, Edema, Fasciotomy, Hypoxia, Snake Bites.

\section{Introduction}

Snakebite is a specialized form of injury that occurs throughout the world, most frequently in the countryside. Depending on the snake species, the injected venom leads to a wide variety of toxic effects, ranging from local tissue damage to systemic organ failure. Anti-snake venom is effective in preventing or limiting the systemic effects. The local symptoms and signs of snake envenomation include severe pain and the appearance of an immobile, tensely-swollen, cold, and apparently pulseless extremity. Clinically, this collection of cytotoxic symptoms can be indistinguishable from concomitantly developing compartment syndrome.

In the literature, however, the management of compartment syndrome in such cases is not clear $[1,2]$. The main objective of this study was to report the very rare case scenario of post-snakebite compartment syndrome involving leg and thigh and to review patient's outcome following snakebite and to highlight the need for undertaking emergency fasciotomy.

\section{Case Report}

A 10-year-old boy presented with alleged history of snake bite by a yellow and black banded krait. Patient was bitten early in the morning, when he was playing outside, on the floor, just in front of his house. He presented in our hospital less than one hour after the bite. At initial clinical examination, there was no bleeding, no neurological abnormalities but the fang marks were clearly visible on his right ankle, just above lateral malleolus. The limb was swollen and tender. Because of the extensive swelling, the child immediately received the first dose of polyvalent anti-snake venom in addition to the usual treatment including tetanus toxoid, tetanus vaccination, amoxicillin, acetaminophen, and ibuprofen and patient was admitted under observation. Despite rapid first aid, the swelling was still increasing and the edema extended from ankle till knee within 12 hours of admission. Patient was anxious, agitated and required increasing dose of analgesia. Paraesthesia, pulselessness and passive stretch pain were positive signs that led 
us to suspicion of compartment syndrome. On day 2, swelling was progressive and extended to the thigh. Swelling and edema failed to diminish even after third dose of anti-snake venom [Fig.1]. The child underwent emergency fasciotomy due to increasing edema and suspected compartment syndrome.

Patient underwent fasciotomy for medial and lateral compartment of leg and for lateral compartment of thigh [Fig.2]. Postoperatively, patient was started on intraveonus vancomycin and meropenem. Subsequently, aspartate aminotranferase (AST) improved following fasciotomy and total leukocyte count started showing falling trend and came to 8970 cells $/ \mathrm{mm}^{3}$ from 21,080 cells $/ \mathrm{mm}^{3}$ on $2^{\text {nd }}$ and $3^{\text {rd }}$ day. Prothrombin time (PT) and international normalized ratio (INR) improved to $17 / 1.10$ from 20.8/1.77 at admission.

Dressing was done every $3^{\text {rd }}$ day under all aseptic precautions under general anaesthesia. Patient was shifted to ward after a couple of days as patient was hemodynamically stable and PT/INR and leukocyte count was showing decreasing trend. Skin grafting was done on $15^{\text {th }}$ day of fasciotomy [Fig.3]. Following skin grafting patient staples were removed after 7 days and patient discharged after 14 days [Fig.4].

\section{Discussion}

Snakebite is a neglected public health problem in tropical and subtropical countries, where rural populations are mainly affected. It is a common occupational hazard mainly in farmers, plantation workers, herders, and laborers leading to significant morbidity and mortality that remains largely unreported. The most affected region in the world is South East Asia because of the dense population and extensive agricultural practices.

India is reported to have the highest number of snake bites $(81,000)$ and deaths $(11,000)$ per year [3]. Fatality due to a snake bite is due to

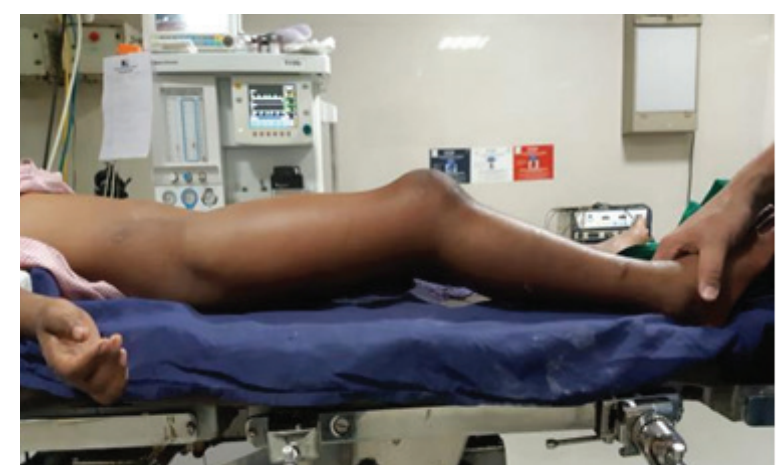

Fig.1: Compartment syndrome of lower limb.
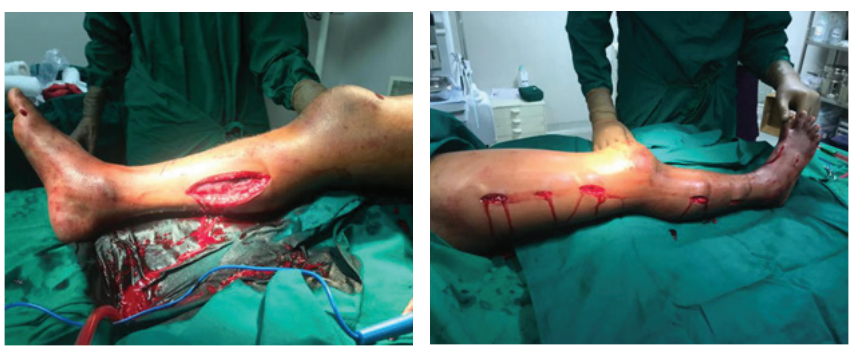

Fig.2: Fasciotomy of lower limb.
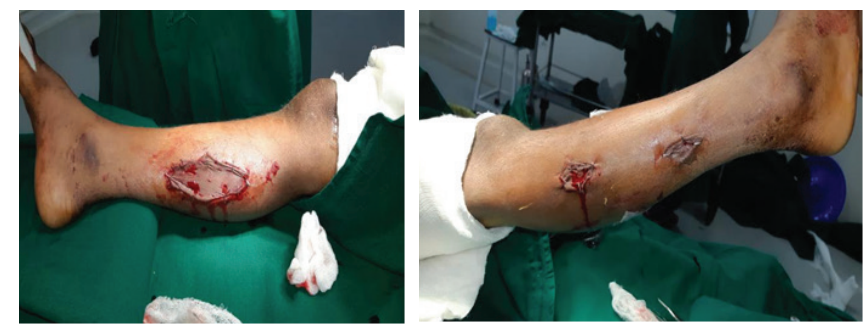

Fig.3: Skin grafting.
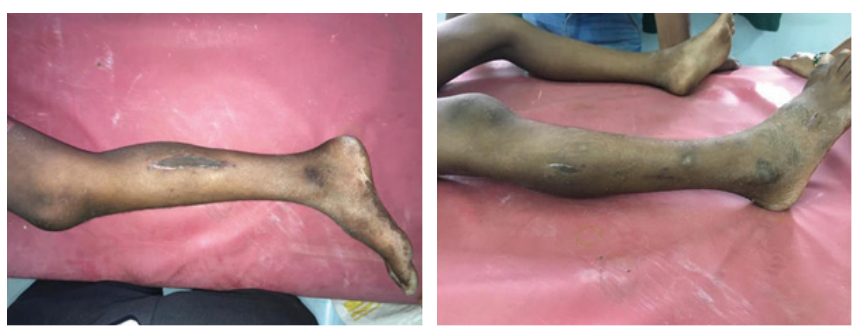

Fig.4: Post skin grafting.

wide species variation, shortage of anti-snake venom (ASV), poor compliance with treatment protocols, lack of public education and clear policy to deal with the problem. Poor access to health care services, difficult transportation, and consequent 
delay in ASV administration results in a high fatality. The time elapsed after the bite is of vital importance because with time more venom gets bound to the tissues and is thus less manageable for neutralization by ASV. Since the development of the venom vaccine, snakebite associated mortality has decreased. There was no mortality in the snakebite patients in this series. Therefore, the management of morbidity after snakebites is the most important consideration [4].

Compartment syndrome is characterized by an increase in intra-compartmental pressure with subsequent neurovascular compromise and tissue necrosis. The clinical signs and symptoms of compartment syndrome include pain, paresthesia, pallor, paralysis, poikilothermia and pulselessness. Several hypotheses have been proposed to explain the development of post-snake bite compartment syndrome (PSCS). Snake venom is composed of a chemically complex mixture that forms enzymatically active proteins after being injected into the human body. After the deposition of venom into subcutaneous tissue, a poisoninduced capillary leak may induce extravasation of plasma and erythrocytes, resulting in edema and ecchymosis. Animal studies have shown that venom deposited intramuscularly causes the release of tissue fluids into the compartment, resulting in elevated pressure.

Close monitoring and compartment pressure measurement are standard approaches in the diagnosis of compartment syndrome. However, not all snakebite patients show immediate signs of compartment syndrome [4]. Furthermore, measuring intra-compartmental pressure in all snakebite patients for the early detection of acute compartment syndrome is impractical. The ischemic signs of compartment syndrome, excluding severe pain, are late signs and should not be relied on for the early diagnosis of compartment syndrome. Therefore, identifying risk factors for compartment syndrome when patients arrive at the ED has become important. In this study, we found that elevated leukocyte count and aspartate aminotranferase were risk factors for the development of PSCS, and further surgery was required.

Proteins and cytokines produced during the acute phase will induce further leukocytosis. In addition, increased AST can be found in skeletal muscle and red blood cells. Acute hemolysis and necrosis of skeletal muscle in severe envenomation may release AST into the circulation. These factors may explain why WBC and AST are useful predictors of PSCS. In symptomatic snakebite patients who have elevated WBC or AST, clinicians should anticipate the development of compartment syndrome. Patients with asymptomatic snakebites are often discharged after being observed for two hours. However, the necessary duration of observation for symptomatic snakebite patients is controversial. In some studies, the authors suggested that symptomatic cases should be monitored for a minimum of $24 \mathrm{~h}$. In our department, to exclude the possibility of compartment syndrome, snakebite patients are carefully observed for local effects at least $48 \mathrm{~h}$ after admission. The result of this case offer valuable insights into snakebites, our results indicate that increased leukocyte count and AST are risk factors for the development of PSCS, our evidence may be insufficient. Therefore, it may not be safe to discharge patients based only on these markers. However, our results may be useful in the development of a decision tool to identify highand low-risk patients in the future. We believe that increases in these markers combined with clinical presentation could be early diagnostic tools and aim to conduct a prospective study to validate these markers.

\section{Conclusion}

Snakebite patients have a high likelihood of developing loco regional complications such as PSCS. Symptomatic snakebite patients should be observed for at least $48 \mathrm{~h}$, and increased leukocyte counts and AST levels are risk factors for PSCS 
[4]. In the future, there may be an opportunity to develop a decision tool that combines observations of clinical symptoms and measurement of leukocyte count and AST levels. Such a tool may be a reasonable and safe way to distinguish patients who can be discharged without needing the 48-h observation period from those who may require surgery.

Contributors: DJ, PM wrote the manuscript and conceptualized the work; TA, AV and AN were responsible for revising it critically. AS, SG and SV provided critical inputs in the manuscript. DJ will act as a study guarantor. All authors approved the final version of this manuscript and are responsible for all aspects of this study.

Funding: None; Competing interests: None stated.

\section{References}

1. Dhar D. Compartment syndrome following snake bite. Oman Medical Journal. 2015;30:1-4.

2. Kim YH, Choi JH, Kim J, Chung YK. Fasciotomy in compartment syndrome from snakebite. Arch Plast Surg. 2019;46:69-74.

3. Gupta YK, Peshin SS. Snake bite in India: Current scenario of an old problem. J Clin Toxicol. 2014;4:1-9.

4. Hsu Chih-Po, Chung Jung-Fang, Hsu Yu-Pao, Wang
Shang-Yu, Fu Chih-Yuan, Yuan Kuo-Ching, et al. Predictors of the development of post-snakebite compartment syndrome. Scandinavian Journal of Trauma, Resuscitation and Emergency Medicine. 2015;23:97.

5. Sanjeev Digra S, Virender Singh V. A clinical profile of neurotoxic snakebite in pediatric population of Jammu region. JK Science. 2016;18:67-70.

6. Meshram RM, Bokade CM, Merchant S, Bhongade S. Clinical profile and outcome of snake bite in children International Journal of Contemporary Pediatrics. 2017;4:910-914.

7. Campbell BT, Corsi JM, Boneti C, Jackson RJ, Smith SD, Kokoska ER, et al. Paediatric snakebites: lessons learned from 114 cases. Journal of Pediatric Surgery. 2008;43:1338-1341.

8. Abbas AD, Abubakar AM, Tahir C. Snake-bite gangrene in children: A report of two cases. Journal of Surgical Technique and Case Report. 2009;1:39-41.

9. Hardy $\mathrm{Sr}$ DL, Zamudio Kelly R. Compartment syndrome, fasciotomy, and neuropathy after a rattlesnake envenomation: Aspects of monitoring and diagnosis wilderness and environmental medicine. 2006;17:36-40.

10. Gras S, Plantefève G, Baud F, Chippaux JP. Snakebite on the hand: lessons from two clinical cases illustrating difficulties of surgical indication The Journal of Venomous Animals and Toxins including Tropical Diseases. 2012;18:467-477. 